\title{
Study of suitability of Fricke-gel-layer dosimeters for in-air measurements to characterise epithermal/thermal neutron beams for NCT
}

\author{
G. Gambarini ${ }^{\mathrm{a}, \mathrm{b}, *}$, E. Artuso ${ }^{\mathrm{a}, \mathrm{b}}$, D. Giove ${ }^{\mathrm{a}, \mathrm{b}}$, M. Felisi $^{\mathrm{a}}$, L. Volpe ${ }^{\mathrm{a}, \mathrm{b}}$, L. Barcaglioni $^{\mathrm{c}, \mathrm{b}}$, \\ S. Agosteo ${ }^{c, b}$, L. Garlati ${ }^{c}$, A. Pola ${ }^{c, b}$, V. Klupak ${ }^{d}$, L. Viererbl ${ }^{d}$, M. Vins ${ }^{d}$, M. Marek ${ }^{d}$ \\ a Department of Physics, Università degli Studi di Milano, Milan, Italy \\ ${ }^{\mathrm{b}}$ INFN, Istituto Nazionale di Fisica Nucleare, Italy \\ ${ }^{\mathrm{c}}$ Energy Department, Politecnico di Milano, Milan, Italy \\ d Department of Neutron Physics, Research Centre Řez,, Czech Republic
}

\section{H I G H L I G H T S}

- Fricke gel dosimeters in form of layers were deeply inspected.

- Suitability of gel dosimeters for characterization of epithermal beams was determined.

- Proper dosimeter shape and dimension were identified.

- Good consistency between measurements and MC simulations was found.

\section{A R T I C L E I N F O}

\section{Article history:}

Received 30 January 2015

Received in revised form

15 June 2015

Accepted 25 July 2015

Available online 28 July 2015

Keywords:

Reactor dosimetry

Epithermal neutron dosimetry

Fricke-gel dosimeter

Monte-Carlo simulation

TLD

\begin{abstract}
A B S T R A C T
The reliability of Fricke gel dosimeters in form of layers for measurements aimed at the characterization of epithermal neutron beams has been studied. By means of dosimeters of different isotopic composition (standard, containing ${ }^{10} \mathrm{~B}$ or prepared with heavy water) placed against the collimator exit, the spatial distribution of gamma and fast neutron doses and of thermal neutron fluence are attained. In order to investigate the accuracy of the results obtained with in-air measurements, suitable MC simulations have been developed and experimental measurements have been performed utilizing Fricke gel dosimeters, thermoluminescence detectors and activation foils. The studies were related to the epithermal beam designed for BNCT irradiations at the research reactor LVR-15 ( $\breve{e}$ ž). The results of calculation and measurements have revealed good consistency of gamma dose and fast neutron 2D distributions obtained with gel dosimeters in form of layers. In contrast, noticeable modification of thermal neutron fluence is caused by the neutron moderation produced by the dosimeter material. Fricke gel dosimeters in thin cylinders, with diameter not greater than $3 \mathrm{~mm}$, have proved to give good results for thermal neutron profiling. For greater accuracy of all results, a better knowledge of the dependence of gel dosimeter sensitivity on radiation LET is needed.
\end{abstract}

(c) 2015 Elsevier Ltd. All rights reserved.

\section{Fundamentals}

Dose measurements in thermal/epithermal neutron fields are challenging due to the complexity of neutron interactions, the very high neutron fluence rates (frequently around $10^{9} \mathrm{~cm}^{-2} \mathrm{~s}^{-1}$ ) and,

\footnotetext{
* Correspondence to: Department of Physics, Università degli Studi di Milano and INFN, Sezione di Milano, via Celoria 16, 20133 Milano, Italy.

E-mail address: grazia.gambarini@mi.infn.it (G. Gambarini).
}

finally, the requirement of separating dose components with different radiobiological effectiveness (RBE).

In tissue exposed to an epithermal neutron field, the absorbed dose components are: (i) the gamma dose, due to the capture reaction ${ }^{1} \mathrm{H}(\mathrm{n}, \gamma)^{2} \mathrm{H}$ and to background from the reactor structural materials, (ii) the 'fast neutron' dose, mainly due to recoil protons produced by elastic scattering of fast and epithermal neutrons with Hydrogen nuclei and (iii) the dose due to protons emitted in the reaction ${ }^{14} \mathrm{~N}(\mathrm{n}, \mathrm{p}){ }^{14} \mathrm{C}$. The photons emitted in capture reactions 
with Hydrogen nuclei have energy of $2.2 \mathrm{MeV}$ and release this energy in the irradiated volume and in normal tissue even in positions far from where the interaction occurs. Then, the spatial distribution of the gamma dose depends on both shape and size of the irradiated volume. The fast neutron dose, in each point, is almost independent of the shape of the irradiated volume and decreases in depth with a distribution depending on the energy spectrum of the beam. The dose due to the protons (about $630 \mathrm{keV}$ in energy) generated from capture reaction with Nitrogen, shows a spatial distribution similar to that of the thermal neutron fluence. The more the epithermal neutrons slow down, the more the direction of their motion becomes isotropic. Therefore, thermalized neutrons diffuse in any direction, also backwards in the volume already passed through. As a consequence, the thermal neutron spatial distribution is dependent on both shape and size of the irradiated volume. Thus the determination of the dose distribution in tissue irradiated in epithermal fields is complex, because all the components of the radiation field should be known, since their RBE is different. In measurements, great attention has to be paid to the composition and dimension of the volumes exposed to the neutron field, in order to avoid altering the distribution of thermal neutrons and the resulting doses.

Currently, the thermal neutron fluence is mostly measured with activation foils or wires, while gamma and fast neutron doses are estimated with paired ionization chambers (Kosunen et al., 1999). Thermoluminescence dosimeters (TLDs) are also utilized for gamma dose mapping, coupled to another detector in order to subtract the contribution of thermal neutrons to their response (Aschan et al., 1999; Burgkhardt et al., 2006). Suitable algorithms to attain gamma dose, with a satisfactory accuracy, from the TL emission curve, avoiding coupled measurements or Monte Carlo (MC) calculations, have been developed too.

In recent years, gel dosimetry has created considerable interest in the neutron capture therapy (NCT) community in particular by exploiting the isotope ${ }^{10} \mathrm{~B}$ (BNCT). Both Fricke and polymer gels have been studied and tested to investigate their suitability for BNCT dosimetry. Polymer gel dosimeters are often preferred, for the higher stability of the dose distribution after irradiation. In fact, in Fricke gel dosimeters ferric ion diffusion causes a loss of resolution of the acquired images and therefore the acquisition of the images must be performed within a relatively short time. In the case of Fricke-Xylenol-Orange gel dosimeters, the diffusion coefficient is dependent on the amount of Xylenol Orange, and is around $1 \mathrm{~mm}^{2} / \mathrm{h}$ (Gambarini et al. 2004a). Even though in neutron fields there are not high dose gradients, as instead occurs in the case of radiotherapy with photons or with charged particles, however it is appropriate to perform the dosimeter analysis within a few hours after irradiation. In order to avoid the trouble of setting up the analysis instrumentation sufficiently near to the irradiation facility, polymer dosimeters have been taken into consideration. Some studies on the suitability of polymer gel dosimeters for BNCT have been carried out, concerning both their tissue equivalence (Wojnecki and Green, 2001) and the potential for dose distribution measurements in NCT neutron fields (UusiSimola et al., 2003, 2007). Such dosimeters have revealed good tissue equivalence, but in epithermal neutron beams they allow only relative dose determinations, because the absorbed dose is the sum of contributions due to different secondary radiation with different linear energy transfer (LET), for which the sensitivity of the dosimeter is different.

FGLDs give the possibility of measuring the spatial distribution of each dose component. In fact, a method for achieving the separation of the different dose contributions has been developed, based on Fricke-Xylenol-Orange gel dosimeters in form of layers, $5 \mathrm{~mm}$ in total thickness and $3 \mathrm{~mm}$ in gel thickness. The separation of the dose components is achieved by analytical comparison of the doses measured with gel dosimeters prepared with or without an isotope whose reaction with neutrons produces charged particles. Images and profiles of boron dose (and also of thermal neutron fluence), are attained by means of two FGLDs, one with the standard composition and the other containing a suitable amount of ${ }^{10} \mathrm{~B}$ (Gambarini et al., 2002, 2004b). Usually, $40 \mu \mathrm{g} / \mathrm{g}$ of ${ }^{10} \mathrm{~B}$ are introduced into the gel composition. In a gel dosimeter containing ${ }^{10} \mathrm{~B}$, the absorbed dose is due to gamma rays, to epithermal and fast neutrons (mainly through recoil protons) and to the charged particles generated by the ${ }^{10} \mathrm{~B}(\mathrm{n}, \alpha){ }^{7} \mathrm{Li}$ reaction. The boron dose is then obtained by pixel-to-pixel elaboration of the dose images measured with a standard dosimeter and a dosimeter containing ${ }^{10} \mathrm{~B}$. The sensitivity of gel dosimeters to the dose due to charged particles is lower than that due to gamma dose. The dependence of the gel dosimeter sensitivity on linear energy transfer (LET) is not well known and needs further research. The coefficient of relative sensitivity to boron dose with respect to gamma dose was recently found to be 0.55 (Gambarini et al., in press), but the uncertainty on this value is still high. This uncertainty is the main source of error in the determination of the boron dose in BNCT and of the thermal neutron fluence that can be evaluated from the boron dose by means of kerma factors. Due to this low sensitivity to charged particles, the boron dose is often a low contribution to the total dose measured with gel dosimeters. With the aim of imaging thermal neutron fluence, therefore, a higher amount of ${ }^{10} \mathrm{~B}$

in the dosimeter composition may seem advantageous, but a higher amount of the chemical compound containing boron gives a great decrease of the yield of ferrous ion oxidation in the dosimeter. For the preparation of the dosimeters with boron, sodium tetraboratetecahydrate $\left[\mathrm{B}_{4} \mathrm{Na}_{2} \mathrm{O}_{7} \cdot 10 \mathrm{H}_{2} \mathrm{O}\right]$ was added to the chemical solution. A compound containing only the isotope ${ }^{10} \mathrm{~B}$ (that is $19.9 \%$ of natural B) would be more advantageous for thermal fluence measurements, but it was not yet used because too expensive.

Fast-neutron and photon doses can be separated by means of a pair of FGLDs, one made with water and the other with heavy water. Fast neutron dose is mainly due to recoil protons in the first gel and to recoil deuterons in the second one. In the algorithms that allow separating gamma and fast neutron doses (Gambarini et al., 2010) the relative sensitivity of gel dosimeters to slow-down protons and deuterons have to be considered. Satisfactory results have been obtained with the present uncertainty on the dependence on LET of the gel dosimeter sensitivity. Further knowledge on this topic will increase the achieved precision.

Concerning the consistency of the results, a better reliability is attained for measurement in-phantom than in-air. For in-phantom measurements, Fricke-gel-layer-dosimeters (FGLDs) are suitable, because of their water equivalence. Moreover, the layer geometry avoids significant variation of neutron transport due to variations of the isotopic composition of the gel matrix.

FGLDs have been utilized many times for in-phantoms measurements, initially at the thermal and epithermal columns of the ENEA's research reactor TAPIRO in Casaccia (It) (Gambarini et al., 2001, 2002) and subsequently at the BNCT epithermal column of LVR-15 research reactor, at the Research Center Řež (Czech Republic) (Burian et al., 2009, Bartesaghi et al., 2009; Gambarini et al., 2010).

Some in-air measurements have also been carried out for gamma and fast neutron dose imaging at the collimator exit of the BNCT epithermal column of the LVR-15 reactor (Gambarini et al. 2011). Other measurements have been performed with moderated neutrons. In fact, for biological experiments on small animals or cell cultures carried out at epithermal columns, the epithermal neutrons are suitably thermalized by means of a layer of moderating material. The moderator chosen for biological experiments at the LVR-15 research reactor consists in a polyethylene disk placed 
at the exit of the epithermal column. Some measurements were carried out to characterize the thermalized neutron beam exiting from the polyethylene moderator (Gambarini et al., 2014a).

Recently, the reliability of the use of Fricke gel dosimeters for in-air measurements and the possible influence of the material constituting the dosimeters on the measured parameters, have been investigated by means of experimental measurements and Monte Carlo simulations. Some results are presented and discussed here.

\section{Materials and methods}

Measurements aimed at investigating the reliability of FGLDs for in-air measurements have been carried out at the BNCT epithermal column of LVR-15 reactor. The collimator exit has circular shape, $12 \mathrm{~cm}$ in diameter.

Some MC simulations and in-air measurements have been carried out at the collimator exit, aimed at the characterization of the epithermal neutron beam. Other MC calculations and experimental measurements have been performed with thermalized neutrons exiting from a polyethylene moderator $2 \mathrm{~cm}$ in thickness and $12 \mathrm{~cm}$ in diameter, affixed at the exit of the epithermal column. In addition to the FGLDs measurements, some tests with TLDs and activation foils have been performed.

\subsection{FGLDs}

The dosimeters are laboratory-made by introducing the dosimetric gel in specially designed containers. In the case of FGLDs, Fricke-gel is held in polystyrene frames of various shapes, $3 \mathrm{~mm}$ in thickness and closed between two transparent polystyrene sheets $1 \mathrm{~mm}$ in thickness. Frames and sheets are kept together with Parafilm ${ }^{\mathbb{R}}$, suitably assembled to minimize the penetration of oxygen into the gel. Two small holes (1 $\mathrm{mm}$ in diameter) in the frame allow introducing in such a structure the prepared solution, before it is gelling. For in-air measurements, circular dosimeters frames have been utilized, with various diameters. In Fig. 1, a sketch of the components of the structure for circular dosimeters and an assembled one are shown.

Dose images have been obtained by means of optical analysis. The absorbed dose, which is related to the difference in optical absorbance, is obtained from pixel-to-pixel manipulations (by using appropriate algorithms) of optical transmittance images acquired by a CCD camera before and after irradiation.

The dosimeter chemical composition, preparation and analysis of the dosimeter are described in previous works.

\subsection{TLDs and activation foils}

In order to attain profiles of both gamma dose and thermal neutron fluence, measurements with TLD-700 chips have been carried out. At thermal-neutron-fluence levels as those of a reactor beam, both photons and thermal neutrons contribute to the response of TLD-700 detectors. In contrast, fast neutrons release only a negligible energy in such dosimeters. In this experiment,

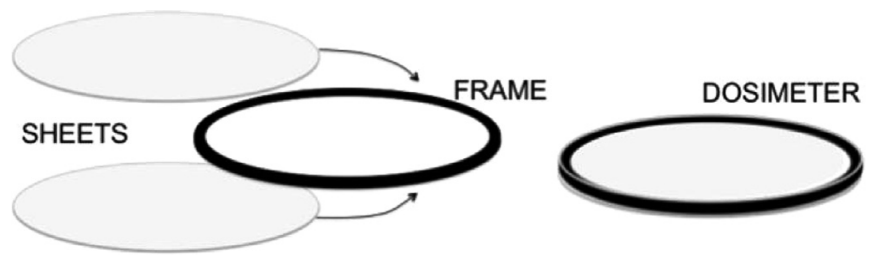

Fig. 1. Structure for FGLD circular dosimeter assembly. the gamma dose and the thermal neutron fluence have been evaluated by analysing the shape of the glow curves of TLDs-700, by applying a method described elsewhere (Gambarini et al., 2014c).

Some TLDs, protected with a thin Teflon film, were inserted in the same dosimeter structure as that shown in Fig. 1 filled of gel without the Fricke solution. In such a way, the values measured with TLDs, carefully calibrated, give information about the doses measured with FGLDs.

For gamma calibration, TLDs were inserted in a suitable waterequivalent phantom and irradiated with ${ }^{60} \mathrm{Co}$ source at uniform dose rate of $0.10 \mathrm{~Gy} / \mathrm{min}$. For calibration against thermal neutrons, TLDs were affixed to a polyethylene disk $2 \mathrm{~cm}$ in thickness, which was inserted at the epithermal column exit. The response obtained after subtraction of the gamma-dose contribution was correlated to the thermal neutron fluence, which was measured with bare and cadmium-covered indium foils in the same irradiation geometry.

\subsection{MCNP simulations}

In this work, MC calculations concerning radiation transport and dose distribution in FGLD exposed to the epithermal neutron beam were carried out. Simulations were performed with MCNP/ MCNPX, 2006. The same file utilized for the treatment planning was used as the radiation source, containing the spectral and spatial characteristics of the neutron source (Burian et al., 2006). In this file, neutrons are emitted by a plane circular source, around the collimator exit, $32 \mathrm{~cm}$ in diameter, with a probability distribution depending on the radial distance from the collimator center. Different configurations have been simulated in MC geometry. Transversal profiles of the fluence of neutrons below $0.5 \mathrm{eV}$ exiting from polyethylene layer of different thicknesses were calculated. The polyethylene disk was placed at the collimator exit. The MC geometry was set in order to investigate the distribution of the three neutron spectral components (thermal, epithermal and fast). In each configuration, the neutron spectral fluence was calculated by using rmesh 1 with the keyword flux. All the obtained tallies are normalized per one source particle.

\section{Results}

MC simulations have been developed to investigate the variation of thermal neutron fluence that can be produced by the $5 \mathrm{~mm}$ of water-equivalent material of which the FGLDs are composed. In a first step, MC calculations have been performed of the fluence of neutrons with energy below $0.5 \mathrm{eV}$ (hereafter called 'thermalized neutrons') exiting from layers of water of different thickness, placed against the collimator exit. In Fig. 2, the transversal central profiles of the thermalized neutron beam outgoing from these layers of water are shown. The profiles were evaluated for thicknesses from 0 to $5 \mathrm{~mm}$. From this figure can be inferred that the thermal neutron fluence of the outgoing beam does not increase markedly. These results, however, are not indicative of the fluence within the FGLD itself. In fact, it should be noted that thermalized neutrons have almost isotropic directions and the thermal fluence in a point of a water-equivalent material placed at the collimator exit depends on the amount of the material after this position and increases with increasing thickness of material. This fact could affect the measured dose and consequently the field characterization. In order to estimate how much this effect influences the dose measurements performed with FGLDs, some MC calculations have been developed. The transversal central profile of the fluence of thermalized neutrons at the central plane of a water disk $5 \mathrm{~mm}$ in thickness, placed against the collimator exit, was then 


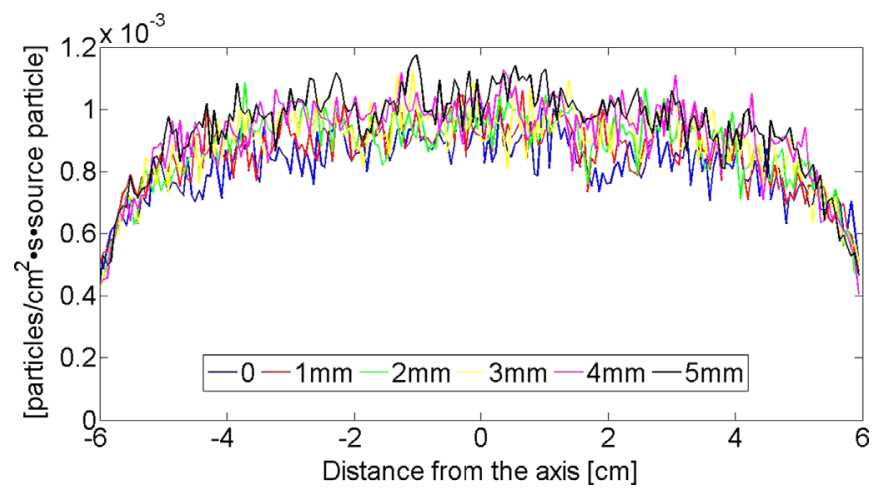

Fig. 2. Transversal central profiles of the thermalized neutron beam outgoing from a disk of water from 0 to $5 \mathrm{~mm}$ in thickness, placed against the epithermal column exit.

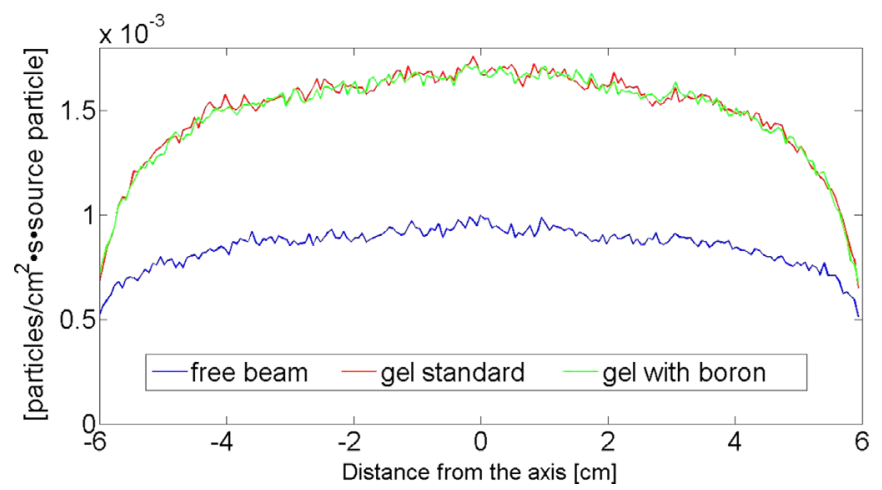

Fig. 3. Transversal central profiles of the fluence of thermalized neutrons at the central plane of a standard FGLD and a FGLD with boron, placed against the epithermal column exit.

calculated. Moreover, to check if the presence of $40 \mu \mathrm{g} / \mathrm{g}$ of ${ }^{10} \mathrm{~B}$ can affect thermal neutron fluence, a similar calculation has been performed in a water disk $5 \mathrm{~mm}$ in thickness with the addition of $40 \mu \mathrm{g} / \mathrm{g}$ of ${ }^{10} \mathrm{~B}$ in the central layer $3 \mathrm{~mm}$ in thickness. The obtained profiles were compared with the transversal central profile of thermal fluence at collimator exit. The results, reported in Fig. 3, show that the contribution of the backscattered neutrons is not negligible but causes drastic increase of the thermal neutron fluence.

This increase in thermal neutron fluence, obviously, has no influence on the fast neutron dose determinations. The influence of this increase of thermal fluence on the gamma dose cannot be predicted. In fact, the consequence is basically an increase in the number of reactions of thermal neutrons with the hydrogen nuclei, but the $2.2-\mathrm{MeV}$ photons generated in such reactions do not release much energy in the gel layer. No MC calculation has been developed about the gamma dose, because the MC simulations evaluate only the gamma dose generated by the neutron beam in the irradiated materials, but the main contribution, in this case, is the reactor background. No experimental measurements were performed at the epithermal exit to verify the correctness of the gamma dose measured with FGLDs. However the results of measurements, above reported, performed at the polyethylene moderator can suggest that the measured gamma dose is not altered by the variation of thermal neutron fluence due to a FGLD.

The results reported in Fig. 3 show also that the addition of $40 \mu \mathrm{g} / \mathrm{g}$ of ${ }^{10} \mathrm{~B}$ in the dosimeter does not cause a significant variation in the thermal fluence and gives a confirmation of the correctness of the method of dose separations by means of FLGDs.

Owing to the interest in the thermalized neutron beams utilized for BNCT biological experiments, MC calculations and

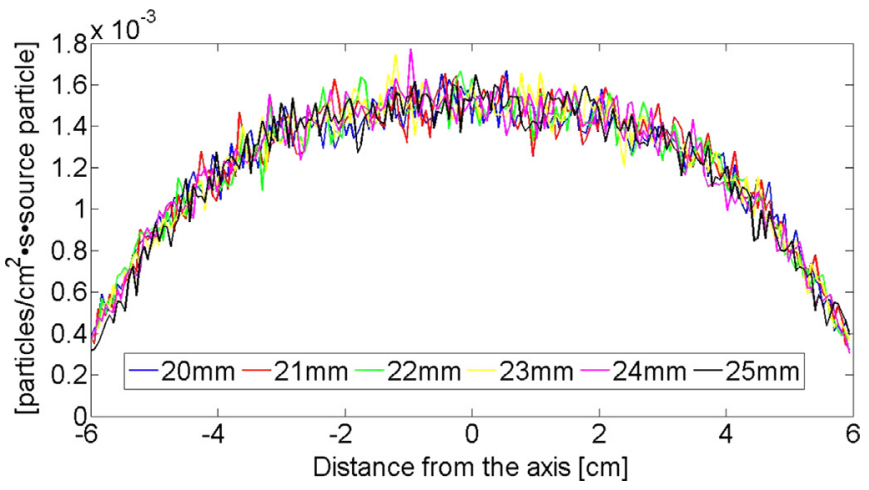

Fig. 4. Transversal central profiles of the thermalized neutron beam outgoing from a disk of water from 20 to $25 \mathrm{~mm}$ in thickness, placed against the epithermal column exit.

experimental measurements were carried out for the beam downstream of the $2 \mathrm{~cm}$ thick polyethylene moderator affixed at the exit of the epithermal neutron beam collimator. In this case, the thermal neutron fluence is high, and can be imaged by means of FGLDs. An initial investigation has been made, by developing MC calculations similar to those related to the unmoderated epithermal beam. Transversal central profiles of the thermalized neutron beam outgoing from a disk of water from 20 to $25 \mathrm{~mm}$ in thickness have been scored. The results are reported in Fig. 4.

Also in this case some MC calculations have been developed to investigate the thermal neutron fluence inside the water volume. The transversal central profiles of the fluence of thermalized neutrons have been calculated, at different depths, in a water disk $5 \mathrm{~mm}$ in thickness, placed against the polyethylene moderator. The results reported in Fig. 5 show a significant increase of thermal neutron fluence from the final edge (corresponding to $5 \mathrm{~mm}$ in the figure) of the exposed material toward the inside. Moreover the results of the same Fig. 5 are coherent with those of Fig. 3. Considering the fact that the result of the measurement made with a FGLD is the average dose in the sensitive volume of the dosimeter ( $3 \mathrm{~mm}$ in thickness) after which there is a polystyrene layer $(1 \mathrm{~mm}$ thickness), it should be emphasized that in the case of dosimeters containing boron the measured dose is that corresponding to a thermal fluence significantly higher than that characteristic of the beam.

Experimental measurements have been carried out to investigate if in FGLDs there is a relevant change of the gamma dose. Some measurements have been performed with TLDs irradiated with two different configurations. One group has been placed against the moderator, along its diameter, and another group has been introduced in a structure similar to that of FGLDs, with a gel

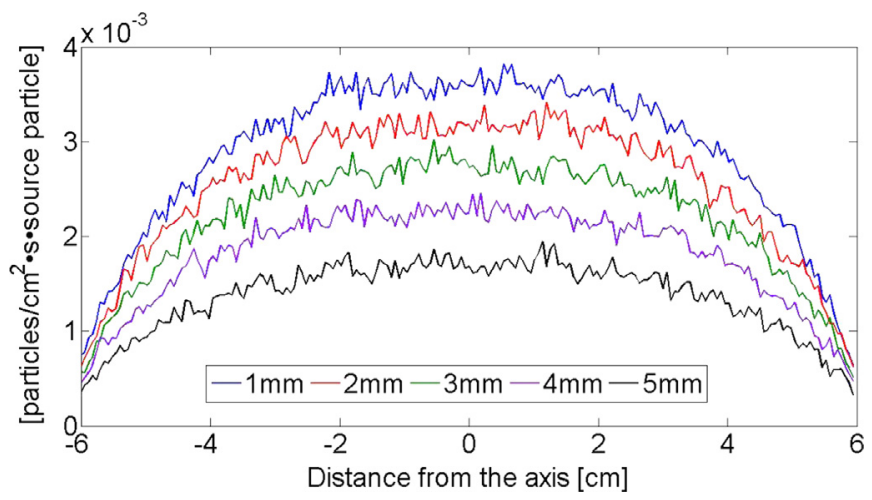

Fig. 5. Transversal central profiles of the fluence of thermalized neutrons calculated, at different depths, in a water disk $5 \mathrm{~mm}$ in thickness, placed against the polyethylene moderator. 


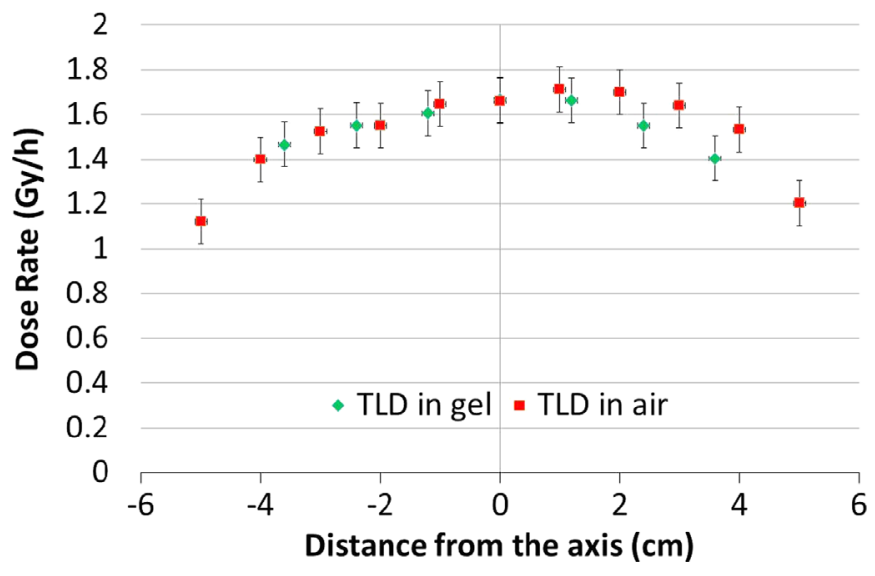

Fig. 6. Gamma doses obtained with TLDs against the polyethylene moderator and with TLDs inserted in the structure imitating FGLD.

matrix not containing the Fricke solution, placed against the moderator. In Fig. 6, the gamma doses obtained with TLDs against the polyethylene moderator and with TLDs inserted in the FGLD structure are reported. These results have proven that the FGLD materials do not cause a change of the gamma dose within experimental uncertainty.

In order to estimate the uncertainty of the measured thermal neutron fluence, boron dose images have been attained by means of a pair of FGLDs with and without boron, placed individually against the moderator and irradiated one at a time. A central profile has been extracted from the boron dose rate image and, by means of kerma factor, the thermal neutron fluence rate profile has been estimated. This profile was then compared with the thermal fluence rate profile obtained by means of the TLDs placed against the moderator, along the diameter. The results are reported in Fig. 7. As expected, from these results it is evident the consistent alteration of the thermal neutron fluence caused by the materials that constitute the FGLDs.

Because of the interest of performing reliable measurements for the characterization of thermal neutron beams, some MC simulations were carried out to evaluate the central transversal profiles of the fluence of thermalized neutrons that would be obtained with gel dosimeters of different shape. In the first step, the effect of reducing the thickness of FGLDs has been considered. In Fig. 8, the central transversal profiles of the fluence of the thermalized neutrons are reported, obtained for FGLDs with thickness from $3 \mathrm{~mm}$ to $5 \mathrm{~mm}$ and, for comparison, the fluence without dosimeters. The advantage of reducing the FGLD thickness seems not very significant and not such as to compensate for the

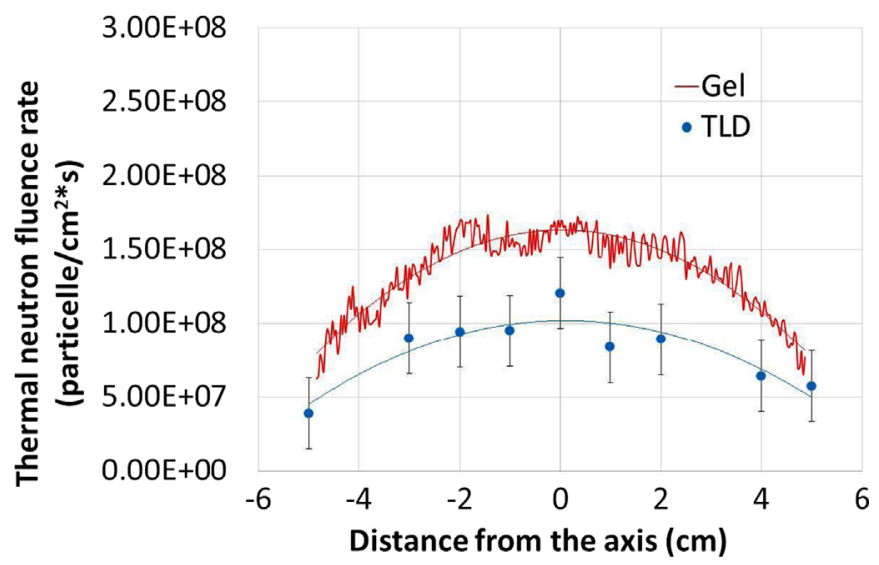

Fig. 7. Thermal fluence transversal profiles after the polyethylene moderator, obtained with FGLDs and TLDs.

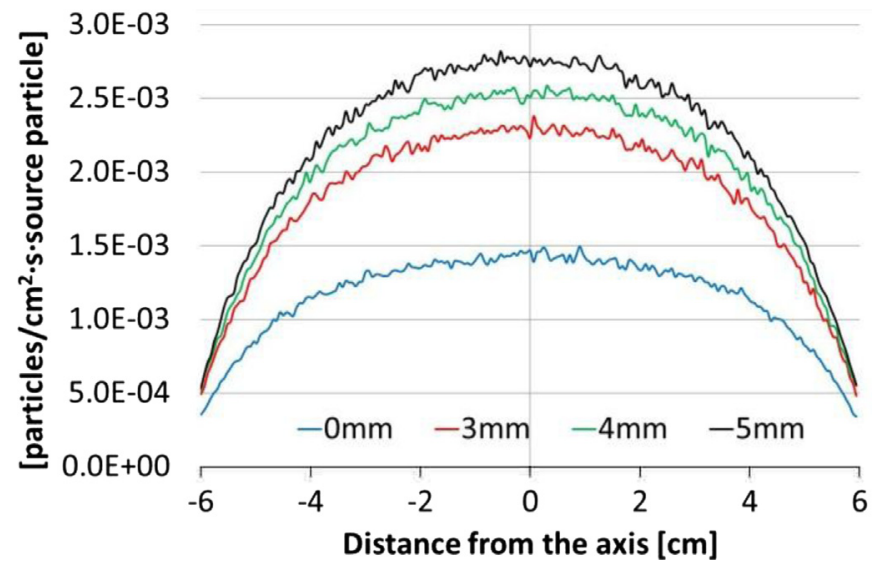

Fig. 8. Transversal central profiles of the fluence of the thermalized neutrons exiting from the polyethylene moderator and that inside FGLDs with different thickness placed against the moderator.

disadvantages that this reduction can give owing to the consequent higher non-uniformity of the gel thickness, greatly increasing the uncertainty of measurements. Therefore, two alternative dosimeter shapes have been considered. The first was the shape of the Fricke gel dosimeters previously proposed for measurements in small phantoms (Gambarini et al., 2014b). These dosimeters are obtained by introducing the Fricke-Xylenol-gel solution, before gelling, in small plastic transparent and not flexible tubes $3 \mathrm{~mm}$ in external diameter. For the MC simulations, these thin tubes were placed against the moderator disk, along its diameter. Another hypothesis was that of utilizing two cuvettes of spectrophotometers, having square section of $10 \mathrm{~mm}$ in side, $45 \mathrm{~mm}$ in length, placed against the moderator one after the other along a diameter, so to give a dosimetric system $90 \mathrm{~mm}$ in length. The profiles of the fluence of thermalized neutrons, obtained in water volumes having these two shapes, are reported in Fig. 9. In the figure, also the profile without dosimeters and the profile in the FGLD $5 \mathrm{~mm}$ in thickness are shown.

From Fig. 9, it is evident that boron dose and thermal neutron fluence can be measured with good accuracy by means of gel dosimeters having the shape of thin cylinders with an external diameter of $3 \mathrm{~mm}$. Paired dosimeters, with and without boron, are required to attain boron dose and then thermal neutron fluence. Particular attention must be paid to the selection of the material with which the cylinders are made. For the optical analysis, the small tubes must be transparent, rigid and with exactly circular section. Commercially it is possible to find small cylindrical tubes with these characteristics. These industrial materials sometimes after irradiation are activated. This is not a serious problem as

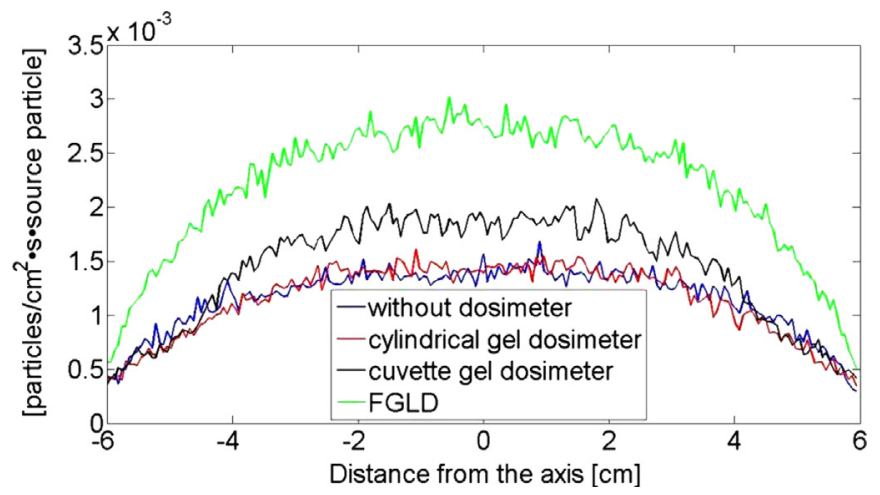

Fig. 9. Transversal central profiles of the fluence of the thermalized neutrons exiting from the polyethylene moderator and that inside dosimeters of different shape placed against the moderator. 
regards the measurement of the boron dose and of the thermal fluence, because the dose due to this activation is the same in the two dosimeters with and without boron, and therefore does not affect the result obtained with the separation algorithms. Obviously, in this case the obtained values of gamma dose (or gamma plus fast neutrons) could be somewhat inaccurate but, as seen before, for such doses it is convenient to utilize FGLDs, because they can give reliable results for these determinations.

\section{Conclusion and remarks}

Considering the results presented above, we can now summarize briefly advantages and limitations of the studied dosimeters.

A general consideration, valid for measurements both in phantom and in air, is that more accurate results could be obtained with a better knowledge of the dependence of the gel dosimeter response by radiation LET. The current lack of information regarding this dependence has influence in principle on all dose determinations, but usually does not cause serious consequences in the determination of the gamma and fast neutron doses, but only in the determination of the boron dose and consequently of thermal neutron fluence.

In phantoms exposed to thermal or epithermal neutrons, the dosimeters having shape of layer, with a total thickness of $5 \mathrm{~mm}$, do not perturb the radiation field and can give satisfactory results for measurements of the spatial distribution of all dose components.

Regarding in-air measurements, aimed at the characterization of epithermal or thermalized neutron beams, FGLDs placed against the beam exit allow satisfactory determination of the spatial distribution of gamma and fast neutron doses. On the contrary, FGLDs give a noticeable perturbation of the thermal neutron fluence in the volume of the dosimeter material. This fact has shown to give consequences only in the determination of boron dose and thermal neutron fluence. To overcome this drawback, a different shape of dosimeter can be chosen for boron dose measurements, as that of a thin cylinder with diameter of about $3 \mathrm{~mm}$.

It is important to underline that the here exposed results and considerations are not significant only for FGLDs. They can be applied directly to gel dosimeters different from Fricke gel, because all these dosimeters have good water-equivalence. Of great importance are the shape and the dimensions of the dosimeters.

Moreover, these results can be useful in order to properly design the materials and supports of other kinds of dosimeters.

In the case of thermal columns consisting in a region inside the reactor structure in which the samples are introduced for irradiation, the thermal neutrons arrive from all directions and so, obviously, these considerations cannot be applied because the situation is completely different. Suitable studies and experiments have to be carried out in order to perform reliable dose measurements in these neutron fields.

In conclusion, despite the current limits, which will be partially overcome, gel dosimeters in form of layer have the exclusive characteristic of being capable of rapidly providing images of dose and fluence. With detectors currently used for BNCT dosimetry, a lot of measurements would be necessary to achieve mappings and then images. To attain accurate results with gel dosimeters, only one measurement with a standard detector, in a chosen position, could be sufficient to normalize the obtained image.

\section{Acknowledgment}

This work was partially supported by the National Institute of Nuclear Physics (INFN, Gr. V, DANTE), Italy.

\section{References}

Aschan, C., Toivonen, M., Savolainen, S., Stecher-Rasmussen, F., 1999. Experimenta correction for thermal neutron sensitivity of gamma ray TL dosemeters irradiated at BNCT beams. Radiat. Prot. Dosim. 82, 65-69.

Bartesaghi, G., Burian, J., Gambarini, G., Marek, M., Negri, A., Viererbl, L., 2009 Evaluation of all dose components in the LVR-15 reactor epithermal neutron beam using Fricke gel dosimeter layers. Appl. Radiat. Isot. 67, S199-S201.

Burgkhardt, B., Bilski, P., Budzanowski, M., Böttger, R., Eberhardt, K., Hampel, G., 2006. Application of different TL detectors for the photon dosimetry in mixed radiation fields used for BNCT. Radiat. Prot. Dosim. 120, 83-86.

Burian, J., Flibor, S., Marek, M., Rejchrt, J., Viererbl, L., Tomandl, I., 2006. Physics for BNCT. J. Phys.: Conf. Ser. 41, 174-186.

Burian, J., Klupak, V., Marek, M., Rejchrt, J., Viererbl, L., Gambarini, G., Bartesaghi, G., 2009. LVR-15 reactor epithermal neutron beam parameters-results of measurements. Appl. Radiat. Isot. 67, S202-S205.

Gambarini, G., Agosteo, S., Danesi, U., Garbellini, F., Lietti, B., Mauri, M., Rosi, G., 2001. Imaging and profiling of absorbed dose in neutron capture therapy. IEEE Trans. Nucl. Sci. 48, 780-784.

Gambarini, G., Birattari, C., Colombi, C., Pirola, L., Rosi, G., 2002. Fricke-gel dosimetry in boron neutron capture therapy. Radiat. Prot. Dosim. 101, 419-422.

Gambarini, G., Birattari, C., Mariani, M., Marchesini, R., Pirola, L., Prestini, P., Sella, M., Tomatis, S., 2004a. Study of light transmittance from layers of Frickex-Xylenol-Orange-gel dosimeters having different composition and analysed with various modalities. Nucl. Instrum. Methods B 213, 321-324.

Gambarini, G., Colli, V., Gay, S., Petrovich, C., Pirola, L., Rosi, G., 2004b. In-phantom imaging of all dose components in boron neutron capture therapy (BNCT) by means of gel dosimeters. Appl. Radiat. Isot. 61, 759-763.

Gambarini, G., Bartesaghi, G., Burian, J., Carrara, M., Marek, M., Negri, A., Pirola, L. Viererbl, L., 2010. Fast-neutron dose evaluation in BNCT with Fricke gel layer detectors. Radiat. Meas. 45, 1398-1401.

Gambarini, G., Bartesaghi, G., Carrara, M., Negri, A., Paganini, L., Vanossi, E., Burian, J., Marek, M., Viererbl, L., Klupak, V., Rejchrt, J., 2011. Imaging of gamma and neutron dose distributions at LVR-15 epithermal beam by means of FGLDs. Appl. Radiat. Isot. 69, 1911-1914.

Gambarini, G., Artuso, E., Burian, J., Klupak, V., Viererbl, L., Marek, M., Agosteo, S., Serino, M., Carrara, M., Borroni, M., d'Errico, F., 2014a. Solid state detectors for dosimetry in the BNCT beam of the LVR-15 research reactor. Radiat. Meas. 71, $513-517$.

Gambarini, G., Negri, A., Regazzoni, V., Magni, D., Nolli, R., Campi, F., Burian, J., Marek, M., Klupak, V., Viererbl, L., 2014b. Methods for dose measurements in small phantoms irradiated at BNCT epithermal column. Appl. Radiat. Isot. 88, $118-124$.

Gambarini, G., Magni, D., Regazzoni., V., Borroni, M., Carrara, M., Pignoli, E., Burian, J., Marek, M., Klupak, V., Viererbl, L., 2014c. Measurements of gamma dose and thermal neutron fluence in phantoms exposed to a BNCT epithermal beam with TLD-700. Radiat. Prot. Dosim. 161, 422-427.

Gambarini G., Artuso E., Giove D., Volpe L., Agosteo S., Barcaglioni L., Campi F., Garlati L., Pola A., Durisi E., Borroni M., Carrara M., Klupak V., Marek M., Viererbl L., Vins M. and d'Errico F., Fricke-gel dosimetry in epithermal or thermal neutron beams of a research reactor, Radiat. Phys. Chem., http://dx.doi.org/10.1016 | j.radmeas.2014.07.004, in press.

Kosunen, A., Kortesniemi, M., Ylä-Mella, H., Seppälä, T., Lampinen, J., Serén, T., Auterinen, I., Järvinen, H., Savolainen, S., 1999. Twin ionisation chambers for dose determination in phantoms in an epithermal neutron beam. Radiat. Prot. Dosim. 81, 187-194.

Uusi-Simola, J., Savolainen, S., Kangasmäki, A., Heikkinen, S., Perkiö, J., Abo Ramadan, U., Seppälä, T., Karila, J., Serén, T., Kotiluoto, P., Sorvari, P., Auterinen, I. 2003. Study of the relative dose-response of BANG- $3{ }^{\mathbb{R}}$ polymer gel dosimeters in epithermal neutron irradiation. Phys. Med. Biol. 48, 2895-2906.

Uusi-Simola, J., Heikkinen, S., Kotiluoto, P., Serén, T., Seppälä, T., Auterinen, I., Savolainen, S., 2007. MAGIC polymer gel for dosimetric verification in boron neutron capture therapy. J. Appl. Clin. Med. Phys. 8, 114-123.

Wojnecki, C., Green, S., 2001. A computational study into the use of polyacrylamide gel and A-150 plastic as brain tissue substitutes for boron neutron capture therapy. Phys. Med. Biol. 46, 1399-1405. 